\title{
El problema del conocimiento en el estudio de la práctica educativa
}

\author{
The problem of knowledge in the study of educational practice \\ José Antonio Castorina \\ Universidad Pedagógica Nacional, Universidad de Buenos \\ Aires, CONICET, Argentina \\ ctono@fibertel.com.ar
}

\section{Resumen:}

Hay suficientes razones para justificar plenamente que los estudios sobre el conocimiento son relevantes para interpretar las prácticas educativas, aunque ello no es reconocido en buena parte de la investigación educativa actual, en nuestro medio. A este respecto, en primer lugar, se formula la pregunta acerca del significado del enfoque de las competencias, argumentando que deja de lado, justamente, la reconstrucción de los objetos de conocimientos durante el aprendizaje escolar. Luego, se discute la fuerte orientación, en muchos trabajos contemporáneos sobre la enseñanza, hacia estudiar la relación entre docentes y alumnos con total ausencia de los saberes que se transmiten en el aula. En tercer lugar, y centralmente, se enfoca la transferencia directa de los resultados de la investigación en diversas disciplinas, en ocasiones con una impronta prescriptiva, sobre los procesos de enseñanza y aprendizaje. Se muestra que dicha transferencia es particularmente significativa en el caso de la psicología del desarrollo y las neurociencias. Además, se sostiene que dicho aplicacionismo ha sido responsable del desconocimiento de la problemática del conocimiento en la investigación educativa actual. Finalmente, se argumenta en defensa de la recuperación de la problemática de los conocimientos en las prácticas educativas, proponiendo una reconfiguración de las unidades de análisis.

Palabras Clave: Práctica Educativa, Objeto de conocimiento, Competencias, Aplicacionismo, Unidades de análisis.

\section{Abstract:}

There are enough reasons to fully justify that studies about knowledge are relevant to interpret educational practice, though this argument is not recognized in great part of this educational research, in our midst. In this respect, first of all, this article poses the question about the meaning of the competence approach arguing that it leaves aside, precisely, the reconstruction of the objects of knowledge during the process of school learning. Secondly, this work argues about the strong trend - in many contemporary works about teaching - to study the relationship between teacher and student without considering the knowledge that is transmitted in the classroom. Thirdly, and most importantly, the article focuses on the direct transfer of the research results about the processes of teaching and learning carried out in different disciplines, sometimes with a prescriptive frame. The work shows that such transfer is particularly significant in the case of developmental psychology and neurosciences. Moreover, the article argues that this application has been responsible of the unawareness of the problems that knowledge poses in the present educational research. Finally, an emphasis in retrieving the knowledge issue in educational practices is presented, proposing, at the same time, a reshaping of the units of analysis.

KEYWORDs: Education practice, Knowledge object, Competences, Applicationism, Analysis unit.

\section{INTRODUCCIÓN}

Al considerar muchos de los trabajos de investigación educativa, en Argentina, tanto los elaborados en Conicet, como en Universidades Públicas o Institutos de Formación Docente, o los presentados en congresos, al menos en los últimos 10 años, se verifica una llamativa disminución de los referidos a los conocimientos en las aulas (Castorina, 2015a). Se tiene la impresión de que las cuestiones vinculadas al proceso de enseñanza y aprendizaje, a la circulación e interacción entre diferentes formas de saber, o a vicisitudes de la apropiación de los objetos de conocimientos socialmente constituidos, tienen un lugar cada vez más restringido. Por el contrario, resulta relevante la problemática del conocimiento, porque la transmisión de los saberes elaborados en la historia de la cultura, desde las matemáticas hasta las 
prácticas corporales, es el propósito básica de la práctica educativa. Y para ello hay que tomar en cuenta los conocimientos adquiridos por alumnos acerca del mundo natural y social, en sus prácticas fuera o dentro de la escuela, y a partir de los cuáles se produce el aprendizaje de aquellos saberes socio-culturales. Así, interactúan las ideas intuitivas de los alumnos y sus creencias como miembros de grupos sociales (representaciones sociales), con los docentes, en los contextos didácticos. En dicho intercambio, participan también los conocimientos de estos últimos sobre cómo realizan sus prácticas de enseñanza, o sus propias representaciones sociales sobre sus alumnos o el sentido de la escuela (Castorina y Sadovsky, 2016) Es, entonces, preciso considerar la intimidad del proceso de reconstrucción de los conocimientos escolares y sus dificultades, la intervención más o menos conflictiva de diversos saberes y conocimientos, o el modo en que maestros y alumnos negocian significados respecto del saber a enseñar. Y no hay dudas de que el éxito en cualquier campo de la educación depende en buena medida de los procesos de transmisión explicita de saberes y de las prácticas socialmente constituidas de producir procesos de enseñanza y aprendizaje, y que ocurren principalmente en el aula (Castorina, 2015a; Castorina y Sadovsky, 2016)

Curiosamente, mientras la institución escolar es objeto de sospechas -cuando no directamente acusadapor los pobres resultados que logra con relación a los aprendizajes de niños y jóvenes, resulta cada vez más escasa en el campo de la investigación educativa la presencia de estudios que abordan la cuestión del conocimiento en las aulas (Castorina, 2015a). En cambio, se puede caracterizar como objetivo de la escuela el trasmitir saberes culturalmente legitimados (en cualquiera de las disciplinas escolares) enmarcada en un proyecto político. De ahí que sea significativo indagar la manera en que los alumnos reconstruyen esos saberes, y establecer las interconexiones de las nociones previas de los alumnos con el saber que se enseña. En este sentido, tales procesos de conocimiento tienen impacto en la calidad educativa, entendida grosso modo, como el aumento de la heterogeneidad y el diálogo entre diferentes saberes, una mayor autonomía en la resolución de problemas, o la actitud crítica de los actores educativos. Aquella reconstrucción está orientada por la intención de enseñar del docente, y se realiza en las interacciones de los alumnos, respecto de un saber social y cultural (Sadovsky y Castorina, en prensa). Para estudiar si se logra o se dificulta la adquisición de los saberes en aula, se requiere apelar a una compleja red de relaciones donde se involucran diferentes dimensiones.

Incluso, asumiendo la perspectiva de las llamadas pedagogías críticas, es razonable pensar la educación más allá de una transmisión de conocimiento, ya que alumnos y maestros se mueven por sus identificaciones, y los compromisos ideológicos, que marcan el proceso de aprendizaje. Por ello, dicha pedagogía apunta a un saber, cuestionador y transformador de la condición de oprimidos de los sujetos, un diálogo que se transforma en una herramienta de liberación (Rigal, 2015). Aun así, sería legítimo preguntarse si la función de la escuela es la de formar directamente sujetos para la emancipación de las condiciones de opresión en nuestra América Latina. En tal sentido, quizás sería pertinente establecer cómo juegan los conocimientos en la escuela, si hay un hiato entre la transmisión de saberes y los conocimientos dirigidos a la sociedad. Quizás aquella actividad con los saberes anclados en las culturas se deba reconocer en relación a la formación de los ciudadanos críticos: la autonomía intelectual y la posición cuestionadora de los alumnos hacia los saberes culturales, posibilitados por los contextos didácticos, autoriza y promueve las propias preguntas acerca de la emancipación, en una interacción democrática en el acceso y validación un saber. En otras palabras, dicha manera de conocer en la escuela habilita al saber liberador, ayuda a pensar la transformación de la sociedad en su conjunto. Por otra parte, en el proceso mismo de la enseñanza se toman inevitablemente posiciones valorativas acerca de los sectores sociales de pertenencia, tanto de alumnos como de docentes. El reconocimiento o el rechazo a la diversidad intercultural, la defensa o la estigmatización de los actores sociales en nuestra propia cultura, se juega durante el proceso de enseñanza y aprendizaje, y contribuye decididamente a democratizar o jerarquizar la adquisición de los saberes escolares. (Novaro, 2010; Llomovate y Kaplan, 2005)

Ahora bien, ni la pedagogía, en sus diferentes perspectivas, ni la psicología constructivista "reconsiderada" (Castorina, 2003) o la versión socio-histórica (Baquero, 2004), o las corrientes neovigotskyanas (Rogoff, 1997; Lave, 2001) ni las didácticas disciplinares (Sadovsky, 2005), hasta la 
sociología de la educación (Bourdieu,1999), ni siquiera el psicoanálisis (Tizio, 2003) para mencionar algunas de las disciplinas, pueden agotar por sí mismas el estudio de aquella trama de relaciones que implica el estudio de la enseñanza y el aprendizaje. Así, sería de mucho interés estudiar a estas últimas, buscando una posible articulación de las relaciones de los alumnos y maestros con el saber, desde la perspectiva sociológica (Charlot, 2007), las vinculaciones de alumnos y maestros con el deseo (Tizio, 2003), desde el psicoanálisis, así como la experiencia con los objetos del mundo socio cultural, como la escritura (Lerner, 2001). A lo dicho se pueden añadir los estudios, en las disciplinas mencionadas, de las dificultades y aún los "fracasos" de algunos alumnos; o la constitución de la subjetividad social de los alumnos en sus relaciones con el saber, con los docentes y con sus pares; indagar para intervenir en las relaciones entre los saberes que se movilizan en las prácticas educativas, tales como los saberes previos de los alumnos, incluidas sus representaciones sociales, y el saber a “enseñar" (Castorina, Barreiro y Carreño, 2010).

De acuerdo a lo anterior, si se acepta la relevancia de los estudios del conocimiento en las prácticas educativas, se vuelven más acuciantes ciertos interrogantes: Por un lado, y habida cuenta de su creciente vigencia en los programas educativos ¿de qué modo el enfoque de las competencias deja de lado la preocupación con el conocimiento?; además, considerando el estado de la investigación educativa, ¿por qué las cuestiones sobre los aprendices y el conocimiento se separan tajantemente de la interpretación de la enseñanza?, más aún, ¿por qué se estudia la relación entre alumno y docente con total ausencia de la participación de los objetos de conocimiento?

Por el otro, en qué sentido una cierta utilización de las diversas disciplinas para el estudio de los procesos de aprendizaje y enseñanza, se puede denominar “aplicacionista”. Particularmente, la transferencia de algunas teorías de la psicología del desarrollo o de las didácticas, sin considerar la especificidad de las prácticas educativas, ha provocado o influenciado fuertemente a la construcción social de aquel "desconocimiento" y el marcado desinterés por estudiar el conocimiento escolar, en el mundo académico (Castorina y Sadovsky, 2016; Bronckart, 2007). En otras palabras, particularmente en las publicaciones y en los congresos de educación, se nota la vacancia de trabajos de aprendizaje de objetos culturales, entre los que se incluyen las prácticas corporales. Esta ausencia ha sido provocada, al menos en parte, por una historia desafortunada de implementaciones disciplinarias (Lerner, 2001; Baquero y Terigi, 1996; Sadovky, 2005; Castorina, 2016) Una aclaración final, las reflexiones que siguen se ocupen de un amplio espectro de prácticas educativas, y las cuestiones de la educación física son tratadas solo episódicamente, por no ser el autor de este campo, aunque quizás algunas de ellas resuenen allí.

\section{LAS COMPETENCIAS}

La introducción de la noción de competencias para promover y evaluar a las prácticas educativas, es,desde un punto de vista histórico, un indicador de que la lógica de la empresa ha comenzado a dominar en la educación, desconociendo que ambos corresponden a temporalidades y significaciones diferentes. Más aún, se ha producido una nítida separación entre los partidarios de homogeneizar la lógica de capacidades y habilidades, asociadas a las demandas del gran capital, y quienes defienden una dimensión socio cultural entre educación y mundo del trabajo (Del Rey, 2012). Entre los primeros, con creciente influencia en la política educativa, el concepto de "saberes" que distribuía y certificaba la escuela fue sustituido por "competencias", por una deslegitimación de los contenidos de saberes y por una justificación de programas orientados por necesidades de éxito a corto plazo de las empresas. De este modo, el interés por la calidad de la educación se apoya en promover las competencias, y con respecto a la práctica de los aprendizajes, facilitando su transferencia y su evaluación. La introducción en el mundo educativo del término "competencia", surgido anteriormente del mundo laboral, se ha resignificado con creciente impacto en las orientaciones de la política curricular y la pedagogía. 
Incluso, para Del Rey (2012) el concepto proviene de un entrecruzamiento de la evaluación cuantitativa de las aptitudes, la educación en términos de mercancía, así como la gestión de recursos humanos. Se propone, entonces, la promoción y evaluación estandarizada de las competencias relevantes, sustituyendo a los programas de conocimientos. Asimismo, al focalizarse en la relación educación y empleo, se rebajan las demandas propiamente educativas al nivel de las exigencias del mercado. Tal orientación forma parte de los programas de los Ministerios de Educación vinculados al neoliberalismo, como hoy es el caso Argentino.

Ahora bien, aunque ésta es una versión hegemónica en el campo educativo, también hay que considerar el sentido que adopta el término en la historia de la psicología, que es otro nivel de análisis, y donde se puede encontrar una doble perspectiva (Bronckart, 2007; 2008). De una parte, Chomsky introdujo la noción de competencia lingüística, en la década de los sesenta del siglo pasado, como una "disposición para el lenguaje", un conocimiento inconsciente de las reglas de transformación, que son innatas y universales. Las producciones verbales son la "realización" de tal competencia teórica, explicando las imperfecciones del lenguaje por factores psicológicos o socio culturales. Esta perspectiva ha impactado en el cognitivismo más duro, volviéndose una de sus categorías centrales, al punto que todas las funciones psicológicas superiores (como la actividad intelectual) se sostendrían en módulos innatos, una especie de competencias ideales semejantes a competencia gramatical.

Por otro lado, para Hymes, (1991) y para Bronckart (2007), las competencias son inseparables de su capacidad adaptativa y contextualizada al mundo social y comunicativo, provienen de alguna forma de aprendizaje. Básicamente, son pensadas como adquiridas en el curso de la acción, se las concibe como procesos mucho más que recursos preexistentes (como en la versión cognitivista). En este sentido, se refieren a las regulaciones por medio de las cuáles los recursos de los individuos son acomodados a los rasgos de las situaciones (Bronckart, 2008).

Hemos mencionado dos enfoques: las competencias innatas y las que derivan de las performances de individuos confrontados a las tareas, por inferencias de cuáles son adecuadas a ellas. Estas últimas -en el campo educativo- serían competencias narrativas, o para calcular, o para resolver problemas sociales. Sin embargo, Hyme (1991), y el propio Bronckart (2007), desconfiaron de ambas perspectivas, y en general de cualquier noción de competencia, por su vinculación a intereses políticos diferentes, y porque son imprecisas en su significado. Con todo, la elección de los contenidos y objetos de la enseñanza se puede realizar, no respecto de capacidades solicitadas al alumno respecto de situaciones, "sino en función de un análisis de los conocimientos prácticos requeridos para participar plenamente en la vida social y comunicativa” (Bronckart, 2007, p.165) Es preciso, a este respecto, integrar al discurso sobre las competencias a categorías como agencia, contexto, acción social y diversidad. Aquellas se adquieren o se construyen en contextos socio-culturales, en lo que podríamos denominar enseñanza situada, en oposición a las competencias universales y genéricas, que se han estudiado descontextualizadas de las situaciones concretas. Quizás la competencia no sea una capacidad para adquirir saberes, sino que es un resultado de un proceso situado de aprendizaje de los saberes fuera y dentro de la escuela.

En síntesis, para nosotros la noción se asocia a la apropiación de los saberes, lo que involucra un cuestionamiento a la enseñanza orientada hacia las "competencias", así como a sus consecuencias para el examen de los aprendizajes de alumnos y la formación docente. Este enfoque re significa la nocióndiferenciándose de las dos versiones-y se aproxima a una posición socio histórica y constructivista. ¿Por qué esta discusión conceptual no es ajena a nuestra cuestión del conocimiento? Básicamente, porque al centrar el aprendizaje en el logro de competencias, sobre todo en el sentido de las competencias vinculadas al mercado de trabajo o la versión psicológica innatista, se coloca en un lugar secundario a los conocimientos que se transmiten en la escuela. Nosotros nos permitimos asumir, con Bronckart (2008), la versión alternativa: las competencias se adquieren durante los procesos de apropiación orientada por los docentes de los saberes que se transmiten en el aprendizaje escolar. Por ejemplo, examinando las capacidades requeridas pensando en los conocimientos prácticos adecuados para una participación adecuada en la comunicación social. En otras 
palabras, las capacidades de los actores educativos se pueden concluir en base a las acciones que hacen en la apropiación de los conocimientos que se transmiten. Se trate de esquemas prácticos o conceptuales, o de las reglas que son pertinentes para resolver determinados problemas, en el campo de la educación física, de las matemáticas o la alfabetización.

Finalmente, no podemos sino compartir las críticas de Crisorio (2006) a la utilización de la noción de competencia, entendida principalmente en su versión asociada con el mercado laboral (sean cognitivas, sociales o tecnológicas). Claramente, se tiende a promover un perfil de profesional ajustado a las demandas de eficiencia, formado en una pedagogía tecnicista de la educación física, con algunos resabios conductistas. Por el contrario, es preciso tender a la apropiación activa y dialógica de la educación corporal, de ésta como una práctica cultural y no solo psicomotriz o de mejoramiento de los rendimientos. Lo que supone, por otra parte, tender a promover la crítica de las condiciones sociales de las prácticas profesionales.

\section{LA ENSEÑANZA Y LAS RELACIONES CON EL OBJETO DE CONOCIMIENTO}

Uno de nuestros interrogantes planteados antes era: ¿la enseñanza separada de los procesos de aprendizaje involucra el abandono del estudio de la apropiación de saberes? Nuestra tesis es que sí, en tanto ha sido entendida como un compromiso entre dos personas: la que detenta algún conocimiento y otra que no dispone de él. Una relación por la que el primer sujeto transfiera lo que sabe -sin especificar medios- a la persona que no sabe (Feldman, 2003). Aunque se sostiene, acertadamente, que los docentes se van constituyendo en las interacciones con los alumnos, no resultan relevantes los vínculos con el objeto de conocimiento durante la enseñanza. $\mathrm{O}$, incluso, se habla de las clases en su transcurrir, o la toma de decisiones de los maestros ante preguntas de los alumnos, pero sin analizar estrictamente la apropiación de los saberes (Litwin, 2016).

Desde una perspectiva constructivista reconsiderada (Castorina, 2003), las cosas se ven de otra manera. Se amplía el concepto de enseñanza, incluyendo la propuesta de problemas a los alumnos, a los fines de que puedan reconstruir los contenidos escolares, el suministrar la información necesaria para dicha reconstrucción, o posibilitar la coordinación de diferentes puntos de vista para alcanzar la resolución de las cuestiones de modo conjunto (Lerner, 2001). No se afirma solo que la enseñanza promueve el aprendizaje, sino que ambos son examinados en el proceso de transmisión del saber social, recurriendo a la interacción entre el sujeto didáctico, el maestro y el "saber a enseñar". Justamente, un artículo nuestro sobre las reglas del fútbol (Castorina, 1995), pedíamos a los profesores de educación física que ejercieran un rol tutorial necesario con los alumnos para "promover" la toma de conciencia de dichas reglas, pero no se nos ocurrió subrayar aquella interacción, donde era clave el saber detentado por los profesores, y su transmisión activa hacia los estudiantes.

En ocasiones, se subrayan los escenarios en que el docente, al tomar la palabra, permite que los otros la recreen, o transformen su significado; o da lugar a la resistencia crítica al pensamiento único, facilitando la emergencia de un sujeto político. Esto es, promoviendo el encuentro con los otros, y articulando el deseo de aprender con el poder de enseñar (Ramos, 2013). Sin embargo, esta posición del docente no se opone a la consideración de los saberes, de forma tal que la investigación educativa pone en relación a la intersubjetividad con los saberes que se enseñan. Nos resulta crucial, a este respecto, que la tesis de promover la resistencia al pensamiento único, se podría sostener y precisar durante el proceso de transmisión del conocimiento en el aula: las producciones propias de los alumnos se darían en la apropiación de lo que se enseña, en la escucha de las voces diferentes que son condición para la reconstrucción de los saberes.

En síntesis, si el estudio educativo se limita a las interacciones del alumno con el docente, sin que el alumno se encuentre con aspectos del saber a enseñar, se dejan de lado los conceptos y los procedimientos de validación de cada disciplina (Sadovsky, 2005) Sin desconocer lo propio de las interacciones intersubjetivas, asumimos que el estudio de la enseñanza de los saberes y su aprendizaje amplía notablemente las posibilidades del sujeto educativo. De modo que indagar el intercambio de los alumnos con su docente a raíz de una 
cuestión propia de la enseñanza, les permite construir significados al interior de la relación triádica, y no solo diádica.

Por lo visto, es al menos discutible considerar que la enseñanza es solo una relación entre docente y alumno, ya que se pierde el sentido de la investigación educativa en el aula, al dejar fuera de sus análisis al proceso de apropiación de los saberes escolares. Al enfocarla como transmisión y el aprendizaje de los conocimientos producidos culturalmente, estamos en el corazón de cualquier actividad educativa. Sin embargo, al estudiar la elaboración de los conocimientos en la escuela, es preciso incluir a las investigaciones que no refieren directamente al conocimiento: las referidas a la institución escolar, o las atinentes al sentido de la subjetividad de los alumnos, o al reconocimiento de la diversidad cultural, hasta el ejercicio del poder y la dominación. En su especificidad, éstos contribuyen a comprender el proceso de enseñanza y aprendizaje, por ejemplo, al proponer una secuencia didáctica, juegan un rol destacado las representaciones sociales de grupos de maestros sobre sus alumnos, según sus rasgos étnicos,o al considerar la violencia simbólica (Bourdieu, 1999; Castorina y Kaplan, 1997) sobre los alumnos, se comprende mejor el significado que le atribuyen al aprendizaje.

Por último, lo afirmado en este parágrafo nos evita concebir que el conocimiento solo sea objeto de las didácticas disciplinares, ya que el estudio de su dinámica y de sus dificultades interpela a todas las ciencias de la educación, desde la sociología, pasando por la antropología, también a las pedagogías. Estamos procurando instaurar una reflexión interdisciplinaria, ya que el modo en que el saber se transmite en la enseñanza y se reconstruye por los alumnos, o la transposición del saber disciplinario en saber escolar, entre otros, no pueden agotarse por el estudio de ninguna disciplina (Garcia, 2006; Castorina y Sadovsky, en prensa; Castorina, 2016)

\section{EL APLICACIONISMO}

Con la finalidad de elucidar la manera en que se utilizan las ciencias de la educación para dar cuenta de los aprendizajes, y cómo ello ha contribuido a evacuar el estudio de los conocimientos en la investigación educativa, hay que examinar "el aplicacionismo" (Castorina, 2003; Bronckart, 2007). Nos referimos a los intentos de transferir de manera directa al campo educativo, con una impronta a veces prescriptiva, los resultados de las investigaciones, obtenidos en otros campos de análisis. Lo fundamental parece residir en omitir que el espacio social de la clase, en el que alumnos y docente interactúan en relación a los saberes transmitidos por la escuela, no puede ser descrito aislando sus componentes, lo que implica un serio riesgo de reduccionismo; análogamente, la escena de la enseñanza y el aprendizaje exige explicitar las múltiples restricciones y condicionamientos que las caracterizan. En general, sobre el campo educativo se ha producido por muchos años una sucesión casi ininterrumpida de procedimientos aplicacionistas, en el sentido señalado. En todos los casos, lo típico es que los investigadores en el campo sociológico, neurocientífico, didáctico o psicológico, utilicen en calidad de "ilustraciones" de las teorías a los procesos educativos. En otras palabras, las hipótesis y procedimientos de las diferentes disciplinas no son desafiados por el modo específico en que se adquieren conocimientos en la escuela, no hay disposición en los investigadores para revisarlos en función del nuevo campo de estudio (Castorina, 2003; Lerner, 2001)

Por otra parte, la perspectiva del aplicacionismo que hemos desarrollado en este trabajo no impide que mencionemos otro modo de encarar la utilización de las psicologías y las neurociencias. En este enfoque, inspirado en la obra de Foucault, tanto las investigaciones psicológicas o su práctica de diagnóstico y tratamiento de dificultades de aprendizaje, como la utilización de ejercitaciones neuronales, contribuyen a poner en juego a los dispositivos de poder. Es decir, estas disciplinas han contribuido, con mucha frecuencia, a elaborar pautas de "normalidad y anormalidad" que han legitimado a las prácticas de disciplinamiento, normativización o instaurado tecnologías del yo, para la infancia, en diferentes momentos históricos. Así, según esta perspectiva, los sujetos de la psicología ya están "intervenidos” por la propia práctica educativa, y es a su respecto que las psicologías han suministrado su credibilidad "científica" a esas prácticas (Baquero y 
Terigi, 1996). Esto es, la observación, regulación y facilitación de una secuencia de desarrollo, por ejemplo, se vuelve el objetivo central de la actividad pedagógica. A veces, el dar una orientación teleológica para el curso de los conocimientos o el cuidado de las "desviaciones" en los comportamientos "esperados".

Se trata de dos niveles diferentes de análisis, pero cuya articulación es perfectamente posible. Es decir: buena parte de los conocimientos psicológicos y neurocientíficos mantienen un carácter estratégico (desde los tests, hasta ciertas ejercitaciones físicas, o empleo de psicologías del desarrollo y la educación, o los "paquetes educativos" basados en neurociencias) y dan sustento a esas prácticas. Pero a la vez, se puede cuestionar epistémicamente al "aplicacionismo" de tales teorías y procedimientos, porque han sido transferidas al mundo educativo sin tomar en cuenta su especificidad epistémica, y por ello son incapaces de resolver los problemas que se plantean en el campo educativo (Castorina, 2015, b).

En esta perspectiva, la llamada Educación Física (Crisorio, 2006; 2013) fue estudiada como un campo de implementación de las psicologías del desarrollo, más o menos maduracionista, o la versión de la integración yoica, en la psicomotricidad conductista. En cualquier caso, sin considerar los significados culturales de los movimientos y ejercicios propuestos. Se afirmó un reduccionismo naturalista o un paralelismo dualista entre lo físico y lo mental, o lo individual respecto de los saberes culturalmente producidos y transmitidos. En todo caso, su dificultades para resolver las cuestiones planteadas da lugar a promover otra visión, comprensiva de "la educación corporal", sostenida en una perspectiva de estudio de prácticas sociales, insertas en la cultura, lo que supone construir nuevos objetos y dispositivos de investigación. En términos generales, diversas disciplinas han sido utilizadas distorsionando o desconociendo la especificidad de estas prácticas, y a la vez fueron y son parte hoy de una biopolítica, que se articula con la lógica de la disciplina "educación física" (desde el saber médico al psicológico). Esto es, mantener la vida en la lógica biológica, por medio del disciplinamiento de los cuerpos, su control y su educación (Paredes, 2010).

\section{El aplicacionismo de las neurociencias}

Ante todo, no se trata de discutir la relevancia de los conocimientos acerca del funcionamiento del cerebro humano respecto de los procesos de enseñanza y aprendizaje. Incluso, es deseable que lo que hoy se denomina neurociencias educativas lleguen a formar parte de la investigación educativa y eventualmente pueden contribuir a resolver algunos problemas de la actividad educativa. Pero el entusiasmo desmedido y acrítico - de propagandistas del mercado de medicamentos y de partidarios de la naturalización de las capacidades humanas- no está fundamentado. Por el contrario, puede decirse que el conocimiento del funcionamiento cerebral es condición necesaria para entender la actividad cognoscitiva de escribir, leer, o hacer cálculos, pero ello no lo convierte en condición suficiente ¿Cuáles son las razones por las que últimamente los propagandistas y los propios neurocientistas entienden que las neurociencias son condiciones necesarias y suficientes para interpretar el aprendizaje escolar?

Básicamente, la verdad o falsedad de las afirmaciones de los neurocientistas respecto de su campo de fenómenos, son patrimonio de estos científicos, pero es preciso examinar las cuestiones conceptuales que están involucradas en su aplicación al campo educativo. Esto es, si se pueden o no identificarla incoherencia de algunas definiciones o las falacias lógicas que ocurren en las argumentaciones de algunos neurocientistas (Bennet, Dennet, Hacker \& Searle, 2007; Hruby, 2012). Así, se puede identificar, entre otros, una confusión lógica en muchas publicaciones, ya que entre el aprendiz y el cerebro hay diversos niveles de análisis, que son salteados apelando solo al "cerebro que aprende". Es un error que se puede considerar, desde el punto de vista lógico, entre otros: cuando atributos o conductas de una totalidad son atribuidas a una de las partes de esa totalidad. Es por completo frecuente que se atribuye la habilidad o el proceso de elaboración de la escritura que es un comportamiento que involucra significaciones e interacciones cognitivas con el objeto "escritura", a la actividad cerebral, que condiciona la actividad psicológica. No hay justificación para reemplazar las explicaciones en términos de apropiación simbólica, intenciones, razones o valores, por explicaciones neurológicas. Carece de sentido atribuir al cerebro los atributos de la totalidad, esto es, cuando el ser humano que en sus interacciones con otros y con los objetos forma sus saberes y aprende. El cerebro 
hace posible que pensemos y creemos significados, pero es un error categorial afirmar que el cerebro piensa o aprende (Davies, 2004).

Además, aquellos neurocientistas otorgan al cerebro humano la capacidad de pensar o aún reflexionar, un reduccionismo que sustituye la vida psicológica por los procesos neurológicos ("el cerebro va a la escuela y aprende"). Se trata de un reduccionismo ontológico, en el que una clase de entidades se presume ser otra clase de entidades, aquí, la mente es presupuesta siendo el cerebro; las diversas clases de conducta humanas - sea social, psicológica o moral- son reducidas a las estructuras y funciones del cerebro (particularmente las estructuras del cerebro). Además, la explicación del funcionamiento de los circuitos neuronales va en lugar de las explicaciones de otro nivel, psicológicas o educativas, en una especie de reduccionismo explicativo. Entre los problemas de esta perspectiva, mencionamos que las descripciones tienden a mirar al comportamiento humano sin considerar las intenciones ni las elecciones en las decisiones humanas. Este enfoque merece un particular análisis crítico respecto de las tesis naturalistas que reducen la vida psicológica a procesos biológicos, lo que lleva a asumir otra posición ontológica, de carácter dialéctico, para plantear los problemas de las relaciones entre neurociencias y educación. (Salas, 2003; Benarós et al, 2010; Churchland, 2005)

Particularmente, se trata de situar las relaciones entre el funcionamiento cerebral y sus condiciones culturales o sociales, en una perspectiva ontológica dialéctica, en lo que podemos denominar una teoría sistémica, que propone una interacción entre el subsistema biológico, el psicológico y el social (García, 2000; Castorina, 2016) Esto pone en evidencia que la discusión sobre la aplicación de las neurociencias al campo educativo supone discutir las tesis filosóficas -inevitables- que se asumen al proponer una cierta transferencia de los resultados de la investigación neurocientífica al campo educativo. Ya sea una concepción del mundo naturalista, ya sea una visión diferente, capaz de articular dinámicamente, en lugar del reduccionismo, los procesos de intencionalidad individual y social comprometidos en el aprendizaje escolar con sus condiciones biológicas.

Finalmente, considerando el enfoque lógico y ontológico, antes mencionado, afirmamos que la transferencia directa de las neurociencias al mundo educativo es paradigmáticamente aplicacionista (Hruby, 2012; Salas, 2003). Principalmente, en el sentido de que no se puede transferir directamente los conocimientos legítimos en el campo del estudio del funcionamiento cerebral a la práctica educativa. Se vuelve a presentar la cuestión de la claridad en la diferenciación categorial y la claridad de las definiciones. Cuando los neuro-científicos educacionales no hacen vigilancia epistemológica sobre sus definiciones y justificaciones, su confusión e imprecisión intelectual es transferida a la propia práctica educativa. Por ejemplo, si no hay cuidado en distinguir cuándo los síntomas, digamos, de la inhabilidad matemática no son evidencia por sí mismos de una inhabilidad neurológica para la matemática. O si se cree que utilizando principalmente las vías de aprendizaje del cerebro, predominantemente visual, auditivo y kinestésico, o la realización del ejercicio físico, se accede al "mundo maravilloso del cerebro", donde reside la clave de una vida mejor. Algo parecido, con sus peculiaridades, podría decirse -quizás- de la concepción de que las dificultades para adquirir esquemas y reglas deportivas, se debería atribuir siempre a una inhabilidad neurológica, a una cuestión de circuitos neurales.

También, si no se diferencia entre la dificultad para leer que puede resultar de un déficit neurológico, de cuándo resulta de una dificultad propiamente pedagógica en el proceso de enseñanza y aprendizaje. De este modo se conspira contra el estudio de las vicisitudes de la apropiación de los conocimientos en sala de clase, al ser ésta sustituida por el estudio de las redes neuronales y por la ejercitación que supuestamente provoca cambios cognitivos. En otras palabras, hay una ilusión en creer que nuestro conocimiento de la plasticidad cerebral puede permitir desarrollar estrategias de enseñanza en cualquier nivel de la enseñanza. En tal sentido, es erróneo evacuar el rol de la política educativa, que debería ayudar a crear condiciones para el aprendizaje orientado por el docente, porque todo ocurre en el mundo natural. De este modo, se imponen enseñanzas del funcionamiento cerebral, junto con paquetes educativos a cargo de neurocientistas, sin la participación docente en definir y elaborar con sus alumnos las trayectorias educativas (Terigi, 2016). 
Como ha sido mencionado, los procesos neurológicos son condición necesaria, pero nunca suficientes para el logro del aprendizaje y la enseñanza. Simplemente, porque tales condiciones necesarias no impiden que haya otras condiciones necesarias, como las condiciones sociales de la actividad educativa, y que incluso éstas se extienden más allá del aula. Pensar que el impacto de la desigualdad social o simbólica sobre el desarrollo depende de una inherente inhabilidad neurológica es equivocada. Y es provocada por la aversión de tantos científicos "serios" a las condiciones sociales y se debe a una manera circular de pensar al interpretar los datos.

En definitiva, rechazamos la "aplicación directa" de las neurociencia a la educación y la elevación de psicólogos cognitivistas y neurocientistas en jueces de la eficacia o calidad de los procesos educativos. Y creemos adecuado proponer un diálogo interdisciplinario, de modo tal que la experticidad del neurocientista debe articularse con los saberes propios de docentes de educación corporal, psicoanalistas, psicólogos y didactas. Lo dicho se basa en la tesis de que los procesos cerebrales están mediatizados por los procesos psicológicos y culturales. Aquellos tienen que ser a su vez examinados desde los enfoques propiamente educativos para tener alguna chance de éxito (Hruby, 2012) No se trata únicamente de una "colaboración", con hegemonía de las neurociencias, sino del respeto irrestricto de los saberes específicos del campo educativo y las ciencias de la educación, a las que no se debe pasar por arriba, o sencillamente eliminar, dando recomendaciones "directas" a los maestros. En este punto, nos movemos en un espacio de disputa filosófica y de posiciones ideológicas, tanto como de una jerarquía de valores éticos y políticos, que quita toda esperanza de un encuentro neutral de disciplinas (García, 2006).

Y lo que no es de menor importancia, dado el propósito de este trabajo, habría que reflexionar acerca de cómo la disminución del interés en los proceso de enseñanza de saberes escolares y su aprendizaje, ha creado a su vez, un terreno fértil para que este rutilante candidato -las neurociencia educativa- ejerza atracción sobre buena parte de los docentes, incluso investigadores en educación. Esto es, tal insuficiencia de los estudios en tales procesos ha dejado un vacío que tienden a ser llenados por estudios neurocientíficos que "prescriben" -injustificadamente- a los docentes como enseñar. Asimismo, lo que decimos es enteramente aplicable a las injustificadas expectativas de la llamada Neurociencia Educativa en educación del movimiento, en el mundo de los profesores (Maureira, 2014). Entre otras consideraciones, sobre la base de una filosofía naturalista y reduccionista, se coloca al concepto plasticidad cerebral, que es válido en el campo de la neurociencia, pero se vuelve el núcleo de la aplicación de esta disciplina en la educación física. Para una posición de educación en prácticas corporales, y no de pura educación física, se está ante una modalidad de aplicacionismo, basado en una concepción del mundo o una ontología cuyos efectos en educación son harto discutibles (Crisorio, 2013) Esta última sirve de sustento ideológico a la alianza de neurociencia educativa con la psicología cognitiva de orientación computacional y una pedagogía promotora de "paquetes de actividades formateadas". En conjunto, la creencia de que la neurociencia educativa es básicamente la única manera científica de estudiar el proceso de aprendizaje, incluso para lograr el incremento no solo de la actividad motriz, sino también -de modo predominante- para optimizar los procesos cognitivos, vía la ejercitación de los movimientos (Maureira, 2014).

Nos preguntamos, por el contrario, y de modo tentativo, si la práctica educativa es un posible lugar de aplicación de las neurociencias no "aplicacionista", o en otras palabras, si se puede abrir un espacio para proponer una genuina actividad interdisciplinaria, que respete las autonomías relativas de las disciplinas, y desde una perspectiva de sistema complejo (Castorina, 2016) o de otro enfoque (Lipina y Sigman, 2011). Si ninguna disciplina, por importante que sea, es suficiente para explicar el proceso de enseñanza y aprendizaje de los saberes constituidos socialmente, es preciso imaginar o esbozar un proceso de indagación colaborativa, muy difícil de concretar, justamente, entre otras razones por la hegemonía de concepciones del mundo que disocian los componentes de la experiencia del aprender, y que proponen una ontología reduccionista (Castorina, 2015a; 2016; García, 2006; Morin, 1998; Boaventura y de Ponte, 2011). 


\section{Las Psicologías del Conocimiento}

Es suficientemente conocido que la psicología, sea la versión cognitiva del proceso de la información o las psicologías constructivistas clásica han sido empleadas como modelos para imaginar situaciones educativas. En ningún caso han asumido los desafíos que provoca el estudio de los conocimientos de los alumnos en sus condiciones didácticas, y se han formulado propuestas educativas sin examinar el aprendizaje de los alumnos en la situación peculiar caracterizada por el encuentro entre sus conocimientos previos con el saber a enseñar y el docente. (Castorina, 2003; Castorina y Sadovsky, 2016)

Así, la mayoría de los ensayos de "pedagogía constructivista" han tenido enormes dificultades para asumir este desafío: se han formulado propuestas educativas sin examinar el aprendizaje de los alumnos en la situación peculiar caracterizada por el encuentro entre sus conocimientos previos con el saber a enseñar y el docente, reunidos por algún contrato didáctico. No tomaron en cuenta que el conocimiento didáctico no deriva de las investigaciones psicológicas, ya que involucra no solo el conocimiento del niño, sino también la naturaleza del conocimiento que se comunica y la acción del maestro; que al ingresar en la relación didáctica, el niño se hace alumno, el conocimiento, relativamente espontáneo es ahora una reconstrucción del saber a enseñar, y el adulto se hace maestro. En tal sentido, las relaciones entre maestro, alumno y saber a enseñar están reguladas por contratos implícitos entre los protagonistas del proceso de enseñanza y aprendizaje (Brousseau, 1998). Según lo dicho, hay que desechar la creencia en la psicología como "la ciencia de referencia" para el planteo de problemas educativos, son principalmente los problemas de la transmisión social de los conocimientos instituidos los que promueven y resignifican las indagaciones psicológicas, por otra parte necesarias (Lerner, 2001; Castorina, 2003; ). En otra perspectiva, también anti aplicacionista, hay esfuerzos en las corrientes actuales del pensamiento psicológico post vigotskyano, por aproximarse al examen del conocimiento en contextos escolares, según una perspectiva situada (Rogoff, 1997; Lave, 2001) En conjunto, constituye un serio desafío epistemológico para las teorías psicológicas el afrontar cuestiones inéditas, desde el conocimiento matemático hasta la educación corporal, y lograr explicar la "génesis artificial" de los conocimientos. En este último sentido, Brousseau (1999), al revisar la conformación de la didáctica de la matemática como dominio de investigación, objeta los intentos aplicacionistas de la psicología cognitiva. En ésta, se inferían los conocimientos matemáticos de los niños a partir de los comportamientos que ponían en acto frente a ciertos dispositivos experimentales, destinados a dar cuenta del pensamiento infantil. Ateniéndose a estos últimos, no se puede explicitar el alcance del aprendizaje, ni las relaciones entre los conocimientos puestos en acto por los niños y la noción matemática transmitida por los docentes, en los contextos didácticos. Lo mismo podría decirse, eventualmente, de las decisiones de los profesores de educación física basadas en investigaciones de laboratorio sobre memoria, desarrollo madurativo, actividad motriz, o funciones ejecutivas. En todos los casos, debe quedar claro que no está en discusión los estudios psicológicos del desarrollo o de las funciones psicológicas realizados fuera de la vida educativa. Más bien, los intentos de inferir a partir de ellos ciertas prescripciones acerca de qué y cómo enseñar. Lo que se necesitan son estudios psicológicos del proceso de aprendizaje que ocurren dentro del contexto didáctico, o de las condiciones específicas de la actividad escolar (Castorina, 2003).

Cuestionando al "aplicacionismo" de la psicología, se ha insistido en que para comprender el conocimiento de los alumnos en el aula es preciso estudiarlo en relación con el saber "a enseñar" y con la actividad del docente. Se reclama, entonces, ir más allá de las habilidades y competencias intelectuales o "físicas" de los alumnos, en una apertura de la investigación hacia los procesos de adquisición de los saberes disciplinares o las prácticas corporales, que se transmiten en la escuela y en contextos didácticos (Lerner, 2001). Se acepta la transmisión dialógica e interactiva de conocimientos culturales, y se rechaza la versión centrada en las elaboraciones puramente espontáneas del alumno, o un aprendizaje sin enseñanza. Claramente, éstas últimas han contribuido al desprestigio de los estudios psicológicos sobre el conocimiento, en el mundo educativo. La actual apelación a estos estudios, en diversas corrientes teóricas, ha dado lugar a una profunda revisión 
conceptual, se propone la investigación psicológica en relación a los saberes que circulan en las aulas; a que sus indagaciones se dirijan a los procesos de aprendizaje referidos a las disciplinas escolares, en los contextos de las interacciones y contratos didácticos. Formulado en estos términos, el análisis de la actividad de alumnos y docentes con los objetos de conocimiento no debería dar lugar ni a la desconfianza ni al desconocimiento por parte de los investigadores en educación. (Castorina, 2015a)

El estudio psicológico propuesto debe articularse con indagaciones que no se limiten a las hipótesis psicológicas elaboradas por fuera de los contextos educativos, ya que ahora las hipótesis psicológicas se refieren al aprendizaje en las aulas, en contextos didácticos, a la génesis "artificial" de los saberes; incluso, a la intervención de las representaciones sociales de los actores en el proceso de enseñanza y aprendizaje (Castorina, 2017). Por lo tanto, son los problemas de la transmisión social de los conocimientos instituidos (y las practicas corporales) y su apropiación activa por parte de los alumnos, los que suscitan las nuevas indagaciones psicológicas sobre el conocimiento escolar. Por supuesto, también son de gran interés los estudios de la participación de los alumnos en las actividades escolares, cuyo centro son las interacciones situadas con docentes en contextos culturales (Rogoff, 1997; Lave, 2001; Lave y Wrangler, 1991) Muy particularmente, la emergencia de la didáctica disciplinar de las matemáticas, en la década de los ochenta, antes mencionada, ha significado una profunda revisión del aplicacionismo psicológico. Aunque esto es así porque propuso un estudio específico de la transmisión y aprendizaje de los saberes, sin embargo el aplicacionismo retorna insistentemente en la práctica educativa, y por parte de los propios didactas. Aunque no haya espacio para un tratamiento cuidadoso de este asunto, sí se puede mencionar-entre otras modalidades - la frecuente utilización de indagaciones didácticas para evaluar las prácticas de la enseñanza de los maestros. Es decir considerarlas según cuánto se acercan o desvían las prácticas educativas de tales propuestas, sin la menor consideración de las razones que han conducido a los maestros orientar de cierto modo a sus prácticas. Se trata de un aplicacionismo casi obvio, "al suponer a la teoría como un ideal de lo que debería ocurrir en la escuela” (Castorina y Sadovsky, 2016)

\section{CONCLUSIONES}

En este trabajo se dan razones para pensar, por un lado, que aquella invisibilización de la problemática del conocimiento ha resultado en buena medida del aplicacionismo de las psicologías del conocimiento, y en menor grado, en cierta utilización prescriptiva de las didácticas en la práctica educativa (Castorina y Sadovsky, 2016). En otras palabras, al promoverse la implementación, particularmente de las psicologías al campo educativo, se ha favorecido la invisibilización del estudio de los procesos de apropiación de los saberes constituidos, mientras los estudio sobre la enseñanza se suelen mantener en los términos exclusivos de relaciones intersubjetivas entre maestros y alumnos. Pero simultáneamente, dicho desconocimiento ha posibilitado un clima favorable en el mundo educativo para la "recepción" de los paquetes educativos basados en las neurociencias y las prácticas educativas basadas en las competencias.

Como consecuencia de lo todo lo dicho, y en defensa de la recuperación de una amplia y difícil problemática de los conocimientos en las prácticas educativas, proponemos una reconfiguración de las unidades de análisis, que involucre la interacción de alumnos y docentes respecto de los objetos de conocimiento. Consideramos imprescindible habilitar una escena donde juegan al mismo tiempo las relaciones de unos y otros con el saber socialmente aceptado, en un cierto momento histórico. También resulta imprescindible que se estudie aquella escena de los conocimientos construidos en las prácticas escolares, o las representaciones sociales de maestros y alumnos respecto del proceso de enseñanza y aprendizaje, tanto como los conocimientos de los docentes sobre sus prácticas, tantas veces ignoradas en la investigación académica. Asimismo, es decisivo considerar que las estigmatizaciones sociales o su rechazo, así como la posición ante la diversidad cultural, en la formación docente y en las actividades de sala de clase, atraviesan aquellos procesos de adquisición cognoscitiva (Novaro, 2010). De ahí que al indagar estos procesos 
se deba buscar unificar dinámicamente, en sus contradicciones, a la construcción de conocimientos en la clase, con las disputas de sentido entre los grupos sociales, con sus relaciones de poder, o con la diversidad de saberes que provienen de diversas culturas (Castorina y Sadovsky, 2016).

Sin duda, la ausencia de una mirada dialéctica ha contribuido a la centración excluyente en los estudios disciplinares que se ocupan de las diferentes dimensiones del proceso de enseñanza y aprendizaje. La investigación de aquella tríada relacional de docentes, alumnos y los objetos de conocimiento supone un diálogo, por ahora solo una promesa, entre aquellas disciplinas, en un proyecto político, así como la indagación acerca de la circulación de los diferentes conocimientos en las aulas. Tal colaboración incluye tanto a la filosofía que elucida el significado de los conocimientos y los marcos epistémicos que condicionan a la investigación y a la orientación de las prácticas educativas, a las psicologías del desarrollo "reconsideradas" para examinar los procesos de conocimiento en sala de clase, como a la sociología, o las didácticas disciplinares, y hasta las neurociencias. Que cada una se arriesgue a reformular sus interrogantes, revisar sus hipótesis e interpelarse unas a otras, lo que no supone eliminar todo límite entre ellas ni su hibridación -lo que merecería una discusión que no pudimos hacer-, sino su articulación dialéctica para estudiar un fenómeno tan complejo como el aprendizaje en educación.

Finalmente, si se me permite, y sin ser un profundo conocedor de la mal llamada "educación física", una síntesis de los análisis de este trabajo parece justificar plenamente nuestra apuesta a la coordinación de diversas disciplinas para pensar los procesos de enseñanza y aprendizaje. Básicamente, porque se vincula con el cuestionamiento de una concepción escisionista, tecnicista, biologista, y aplicacionista de ciertos estudios disciplinares respecto del aprendizaje y la enseñanza, y con la afirmación de una perspectiva dialéctica e interdisciplinaria. En este sentido, un brevísimo comentario a las ideas de Crisorio (2013; Crisorio \& Celles, 1999), que nos resultan renovadoras al proponer una educación de las prácticas corporales. Independientemente de algunas tesis que nos atreveríamos a discutir respetuosamente, en otro lugar, y que afirman la "esterilidad" de toda teoría del aprendizaje, o de cualquier teoría del desarrollo del conocimiento, o de admitir solamente al sujeto lacaniano, como el único sujeto de la educación, hay acuerdos básicos, que me permito mencionar, muy modestamente. Al devenir la educación física una educación corporal, se postula el establecimiento de una relación del sujeto con su cuerpo, pero en el contexto de sus vínculos constitutivos con la cultura, de un énfasis en los significados de las diferentes prácticas de cuerpo y mundo social. Este enfoque no reduce las prácticas corporales a ejercicios calisténicos, ni mucho menos a procesos neurofisiológicos, que les son correlativos, pero no determinantes de aquellas. De ahí que al entender a esas prácticas como inseparables del mundo simbólico, se hace una contribución para estudiar la transmisión y el aprendizaje de aquellas prácticas corporales (Crisorio, 2006; 2013). En nuestra opinión, solamente un diálogo abierto, situado en un marco epistémico compartido de una ontología antiescisionista, crítica del dualismo y el reduccionismo, de corte relacional y dialéctico, puede dar lugar a una actividad colaborativa entre las disciplinas mencionadas, o más particularmente a ciertas corrientes de las disciplinas, que comparten aquel marco. En este sentido, algunos argumentos que hemos desplegado, pueden tener -quizás- algún interés indirecto para pensar la formación docente y la orientación de las clases a los alumnos del sistema educativo, en lo relacionado con educación corporal. $\mathrm{Al}$ menos, en lo referido a la apropiación activa de los saberes que se transmiten en la sala de clase, mediante el diálogo de los actores educativos, cuyo estudio no puede ser patrimonio de una sola disciplina, por relevante que sea.

\section{BibLiografía}

Baquero, R (2004) Analizando las unidades de análisis. Los enfoques socio-culturales y el abordaje del desarrollo y el aprendizaje escolar. En J.A Castorina y S. Dubrovsky (Comp) Psicología, Cultura y Educación 67-80. Buenos Aires. Miño y Dávila 
Baquero, R y Terigi, F (1996) Constructivismo y modelos genéticos. Notas para redefinir el problema de sus relaciones con el discurso y las prácticas educativas. Enfoques Pedagógicos, serie Internacional, 12, 4(2)

Benarós, S; Lipina, S; Segretim, S; Hermida, J \& Colombo, J (2010) Neurociencia y educación: hacia la construcción de puentes interactivos, Revista de Neurología, (3): 179-186

Bennet, M. R; Dennet, D; Hacker, P \& Searle, J (2007) Neuroscience \& Philosophy: Mind, Brain and language. New York: Columbia Universtity Press

Boaventura, A. M \& de Ponte, J.P (2011) Investigación colaborativa: potencialidades y problemas. Revista de Educación y Pedagogia, Universidad de Antioquia, 23 (59), 135

Bourdieu, P (1999) Meditaciones Pascalianas. Barcelona. Anagrama

Bronckart, J.P (2008) ¿Es pertinente la noción de competencia en educación?, en Novedades Educativas, 11, 4-9

Bronckart, J.P (2007) Desarrollo del lenguaje y didáctica de la lenguas. Buenos Aires. Miño y Dávila.

Brousseau, G (1998) Théorie des Sutuacions Didactiques. Grenoble. La Pensée Sauvage

Castorina, J.A (2017) Las representaciones sociales y los procesos de enseñanza y aprendizaje de los conocimientos sociales. Psicologia da Educacao. Sao Paulo, 44, 1-33

Castorina, J.A (2016) La relación problemática entre Neurociencia y educación. Condiciones y análisis crítico. Propuesta Educativa, 12 (46), 26-41

Castorina, J.A (2015a) Los problemas del conocimiento escolar en la investigación educativa. Un análisis crítico. Espacios en Blanco. 25.373-392

Castorina, J.A (2015b) Problemas epistemológicos de la investigación en psicología educacional: las prácticas discursivas, análisis meta teóricos y valores. En N. Abate y R. Arué (compl) Cognición, aprendizaje y desarrollo. 23-52. Buenos Aires. Noveduc

Castorina, J.A (2003) Las epistemologías constructivistas ante el desafío de los saberes disciplinares, Psykhe, 12 (2), $15-28$

Castorina, J.A y Sadovsky, P (2016) El significado de los conocimientos en los procesos de enseñanza y aprendizaje. Documento de Trabajo del Seminario Homónimo desarrollado en la UNIPE. Buenos Aires

Castorina, J.A; Barreiro, A \& Carreño, L (2010) El concepto de polifasia cognitiva en el estudio del cambio conceptual, en M. Carretero y J.A. Castorina: La Construcción del Conocimiento Histórico. Buenos Aires. Paidós, 131-152.

Castorina, J.A (1995) La formación de las ideas infantiles sobre las reglas del fútbol: algunos problemas cognitivos. Revista de Educación Física y Ciencia, 1 (1), 7-20

Charlot, B (2007) La relación con el saber. Buenos Aires. El Zorzal

Churchland, P. M (2005) Cleansing Science, Inquiry, 48 (5), 464-477

Davis, A (2004) The Credentials of Brain-Based Learning, Journal of Education, 28 (1), 21-35

Crisorio, R.L (2013) Educación Corporal, Cadernos de Formacao. RBCE.9-19.

Crisorio, R.L (2006) Saber y ser competente no es lo mismo. Educación fisica y deporte. Universidad de Antioquia, 25 (2). $94-103$

Crisorio, R. L \& Celles, M (1999) Apuntes para una didáctica de la educación física en Tercer Ciclo CGB. Revista de Ciencia y Educación Física, UNLP, 75-81

Del Rey, A. (2012) La competencias en la escuela. Una visión crítica sobre el rendimiento escolar. Buenos Aires: Paidós.

Feldman, D (2003) Enseñanza y Escuela. Buenos Aires. Paidós

García, R (2000) El Conocimiento en Construcción. Barcelona. Gedisa

Hruby, G.G (2012) Three requirements for justifying an educational neuroscience, Educational Psychology, 821 (1), $1-23$

Hymes, D.H (1991) Vers la competence de communication. Paris. Crédif-Hartier

Lave, (2001) Las prácticas del aprendizaje. En E. S. Chaiklin y D. Lave (Comps) Estudiar las prácticas. Perspectivas sobre la actividad y el contexto. Buenos Aires. Amorrortu. 
Lave, J \& Wrangler, E (1991) Situated Learning, peripherial participation. New York. Cambridge

Lerner, D (2001) Dialéctica y Psicología. Una perspectiva epistemológica. En J.A. Castorina (Comp) Desarrollos y Problemas en Psicología Genética. Buenos Aires. EUDEBA.

Lipina,S \& Sigman, M (2011) Introducción. Oportunidades y desafíos en la articulación entre la neurociencia, la ciencia cognitiva y la educación, en S. Lipina \& M. Sigman (Eds.) La Pizarra de Babel. Buenos Aires. Libros del Zorzal. 9-24

Litwin, E (2016) El Oficio de Enseñar. Buenos Aires. Paidó

Llomovate, S \& Kaplan, C (2005) Desigualdad Educativa. Buenos Aires. Novedades Educativas.

Maureira, F (2014) Principios de Neuroeducación Fisica. Actividad física, psicología y Neurociencia. Madrid. Ed. Academia Española

Morin, E (1998) Introducción al pensamiento complejo. Barcelona. Gedisa

Novaro, G (2010) (Comp.) Indigenas y migrantes. Buenos Aires. Biblos.

Paredes, F (2010) Educación física Deportiva, Revista Digital, 8 (151).

Ramos, M (2013) Hablemos de enseñanza, en M. Caldo; E. Martinchuk \& M. Ramos (Coords) La infancia en la miradadel@sMestr@s.Buenos Aires.La Crujía.

Rigal, L (2015) Educación Popular: Tensiones y Desafíos, Para Juanito, 3 (8), 48-56.

Rogoff (1997) Los tres planos de la actividad socio cultural: apropiación participativa, participación guiada y aprendizaje. En J. Wertsch, P. del Río y A. Alverez (Eds.) La mente socio cultural. Aproximaciones teóricas y aplicadas. Madrid. Funcación Infancia y Aprendizaje. 11-22.

Sadovsky, P (2005) Enseñar matemáticas hoy. Buenos Aires. El Zorzal

Sadovsky, P \& Castorina, J.A (en prensa) La intervención de los valores en la investigación en didáctica de las matemáticas, El significado de conocimientos y saberes en los procesos de enseñanza y aprendizaje. Buenos Aires. UNIPE

Salas, R (2003) ¿La educación necesita realmente de las neurociencias. Estudios Pedagógicos. 29, 155-171

Terigi, F (2016) Sobre el aprendizaje escolar y neurociencias, Propuesta Educativa, 12 (46), 50-64

Tizio, H (2003) La posición de los profesores en los aparatos de gestión del sistema. En Tizio, H (Coord) Reinventar el vinculo Educativo. Aportes de la Psicología Social y el Psicoanálisis. Barcelona. Gedisa 